\title{
Discours
}

Revue de linguistique, psycholinguistique et

informatique. A journal of linguistics, psycholinguistics and computational linguistics

$20 \mid 2017$

Varia

\section{Changement de topique et prise en charge énonciative : le rôle de toujours est-il que}

\section{Véronique Lenepveu}

\section{OpenEdition}

Journals

Édition électronique

URL : http://journals.openedition.org/discours/9272

DOI : $10.4000 /$ discours. 9272

ISSN : 1963-1723

Éditeur :

Laboratoire LATTICE, Presses universitaires de Caen

\section{Référence électronique}

Véronique Lenepveu, «Changement de topique et prise en charge énonciative : le rôle de toujours est-il que », Discours [En ligne], 20 | 2017, mis en ligne le 22 septembre 2017, consulté le 20 avril 2019. URL: http://journals.openedition.org/discours/9272 ; DOI : 10.4000/discours.9272 

Revue de linguistique, psycholinguistique et informatique

\title{
Changement de topique et prise en charge énonciative : le rôle de toujours est-il que
}

\author{
Véronique Lenepveu \\ CRISCO \\ Université de Caen Normandie
}

Véronique Lenepveu, «Changement de topique et prise en charge énonciative: le rôle de toujours est-il que», Discours [En ligne], 20 | 2017, mis en ligne le 22 septembre 2017.

URL: http://discours.revues.org/9272 



\title{
Changement de topique et prise en charge énonciative : le rôle de toujours est-il que
}

\author{
Véronique Lenepveu \\ CRISCO \\ Université de Caen Normandie
}

L'article fait suite à un premier travail consacré à une analyse aspectuelle et diachronique de la locution adverbiale toujours est-il que à partir de Frantext (Lenepveu, 2015), dans le prolongement des travaux de Hansen (2004) et Buchi (2007). Si la locution indique fondamentalement le retour à un topique discursif antérieur (Van Dijk, 1976; Fraser, 2009a et b) après une séquence «intercalée», simultanément, elle signale la plus forte prise en charge par le locuteur du segment de texte qu'elle introduit. Cette différence de prise en charge énonciative (Dendale et Coltier, 2005) donne lieu à deux emplois distincts de la locution, selon que la séquence intercalée soit l'expression d'une incertitude, ou qu'elle se présente sous une modalité assertive catégorique. L'existence de deux emplois est ici tout d'abord expliquée par une étude sémantique des formes spécifiques prises par la séquence intercalée, puis, dans un deuxième temps, confirmée par une recherche des équivalents possibles de la locution toujours est-il que en anglais dans des traductions (français/anglais; anglais/français) de textes littéraires du XIXe et du XXe siècle. II apparaît que toujours est-il que peut être l'équivalent en anglais d'un marqueur de modalité épistémique (it is certain that, certain it is that) lorsque la fin de la séquence intercalée exprime une incertitude.

Mots clés: locution adverbiale, topique discursif, structure discursive, prise en charge, modalité

This article follows a first paper (Lenepveu, 2015) which provided an aspectual and diachronic analysis of the French lexicalized expression toujours est-il que in continuation of Hansen (2004) and Buchi (2007). It was shown that the lexicalized expression marks a return to the main discourse topic (Van Dijk, 1976; Fraser, 2009a and b) and simultaneously that it reinforces the speaker's commitment. The present paper deals with two distinct uses of the locution: a study of the sentences preceding the locution makes it possible to explain in which contexts toujours est-il que indicates the speaker's high commitment (Dendale \& Coltier, 2005) towards the propositionnal content's validity or towards the content's relevance. Then, a search of English synonyms in translations of litterary texts (French/English; English/French) of the 19th and the 2oth century confirms the analysis. It appears that toujours est-il que is translated by an epistemic modality marker (it is certain that, certain it is that) when it indicates a difference of degree of belief.

Keywords: French locution, discursive topic, discourse pattern, speaker's commitment, modality

\section{Introduction}

Cette recherche s'inscrit dans le cadre d'un projet Labex TransferS (M. Charolles, UMR LATTICE) qui vise à étudier les marqueurs de changement de topique de discours ${ }^{1}$ dans une perspective comparative inter-langues et/ou diachronique. Elle

1. Ce travail a été réalisé avec le soutien du laboratoire d'excellence TransferS (programme «Investissements d'avenir» ANR-Io-IDEX-oooI-02PSL* et ANR-Io-LABX-oo99) au sein du projet «Marqueurs de changement de topique de discours» (http://transfers.ens.fr/articlers5.html). Je remercie vivement les 
fait suite à un premier article consacré à l'analyse aspectuelle et diachronique de la locution adverbiale toujours est-il que et à son rôle dans la répartition des informations dans le texte (Lenepveu, 20I5). Dans le prolongement des travaux de Hansen (2004 et 2008) et Buchi (2007), nous avions posé que la locution toujours est-il que marquait fondamentalement et systématiquement un changement de topique de discours (Van Dijk, 1976; Fraser, 2009a et b; Charolles, 2016) ${ }^{2}$ ou plus exactement, un retour à un topique antérieur, après une séquence dite «intercalée», comportant typiquement l'expression d'une incertitude, voire un aveu d'ignorance. Ainsi, dans l'exemple [I], le jugement de valeur négatif porté sur le goût des vieilles choses se trouve repris comme topique discursif ${ }^{3}$, après une série de questions manifestant l'incertitude du locuteur quant aux raisons motivant ce goût du vieux:

[I] Le goût des vieilles choses est une manie de notre temps. [...] respect condescendant pour l'armoire d'une grand-mère qui n'est pas toujours la nôtre? Ou désir de transformer notre salon en musée pour notre satisfaction personnelle ou l'ébaubissement des visiteurs? Ou snobisme du jour qui nous impose d'avoir un vieil appareil téléphonique à support de bois pour notre standing? Ou arrière-pensée de spéculation? Ou je ne sais quoi. Toujours est-il que le goût du vieux est un mal moderne.

(Frantext: P.-J. Hélias, Le cheval d'orgueil [1975], Paris, Plon, 2008, p. 526)

Mais il n'y a pas nécessairement relation paraphrastique entre les deux segments de texte mis en gras, et la séquence intercalée n'est pas nécessairement l'expression d'une incertitude ou d'une ignorance. Ainsi, dans l'exemple [2], extrait d'un texte de Queneau, toujours est-il que introduit un événement qui s'inscrit à la suite de l'événement décrit dans le premier segment de texte mis en gras, tandis qu'un jugement de valeur à portée générale concernant les zazous constitue la séquence intercalée et s'interprète comme une digression (Hansen, 2004). Toujours est-il que indique la reprise de la narration, le topique du discours étant de nouveau le comportement d'un individu particulier rencontré dans l'autobus:

[2] Cette fois-là je n'avais dans mon voisinage que des hommes, dont une sorte de zazou au col démesuré et qui portait autour de son feutre mou une espèce de tresse au lieu de ruban. Comme si on ne devrait pas envoyer tous ces gars-là dans des camps de travail. Pour relever les ruines par exemple. Celles des anglo-saxons surtout. De mon temps on était camelot du roy, et pas swing. Toujours est-il que ce garnement se permet tout à coup d'engueuler un ancien combattant, un vrai, de la guerre de 14-18. Et ce dernier qui ne riposte pas!

(Frantext: R. Queneau, Exercices de style [1947], Paris, Gallimard, 1963, p. 130)

participants de ce projet pour leurs commentaires et suggestions. Je remercie également les deux relecteurs anonymes, les erreurs qui subsistent sont bien sûr de mon fait.

2. Sur la place réservée aux marqueurs de topicalisation ("Topic Orientation Markers») chez Fraser (20ogb), nous renvoyons à la présentation de $\mathrm{M}$. Charolles des différentes taxinomies proposées concernant les marqueurs de discours en général (diaporama consultable sur la plateforme du projet: http://I29.199.228.Io/pydio).

3. Le contenu asserté et réaffirmé figure en gras dans les exemples. 
La notion de topique discursif, définie comme étant ce dont il est question dans le discours, permet de dégager une structure discursive comprenant:

I. un point de vue initial sur un topique discursif («point de vue» au sens de Nølke [1994]) ${ }^{4}$,

2. une séquence intercalée contenant typiquement l'expression d'une incertitude, voire un aveu d'ignorance, mais aussi un jugement de valeur ou encore un commentaire métadiscursif ${ }^{5}$ interprété comme une digression,

3. le retour au topique discursif initial, retour qui se donne sous la forme d'une reprise du point de vue initial (exemple [I]) ou sous la forme d'une reprise de la narration (exemple [2]).

Selon l'hypothèse de Martin (1987: 12I-I22), adoptée également par Buchi (2007), le fonctionnement énonciatif et textuel de la locution peut s'expliquer à partir de la valeur aspectuelle de persistance, et non de permanence, de toujours, laquelle valeur est transposée sur l'axe de dicto: l'adverbe toujours pris dans la locution ${ }^{6}$, ne porte plus sur le contenu mais sur la prise en charge de l'assertion. Dans l'exemple [I], toujours est-il que signale que le point de vue initial persiste par-delà les incertitudes, tandis que dans l'exemple [2], la locution signale la persistance de l'objet même du discours, c'est-à-dire le maintien du topique discursif. L'étude de l'évolution de la formation de la locution à partir de Frantext ${ }^{7}$ (Hansen, 2004; Buchi, 2007; Lenepveu, 2015) confirme cette analyse et permet de rattacher la valeur de dicto de la locution à la valeur de persistance de toujours, pour affirmer que dans tous les cas, il y a retour à un topique antérieur. De même, l'examen en synchronie des contextes gauches très larges de la locution, à partir toujours de Frantext, semble vérifier l'existence de cette structure discursive. On a alors posé l'hypothèse que, simultanément, la locution sert à préciser le statut énonciatif de la séquence intercalée relativement au segment de texte introduit par toujours est-il que. Nous avons considéré que dans tous les contextes, toujours est-il que signale la plus grande pertinence (Blumenthal, I980: 126-I27) de

4. Dans la perspective polyphonique de Nølke (1994), un point de vue comprend un contenu propositionnel et un jugement portant sur ce contenu, le jugement consistant en la validation, éventuellement modalisée, de la proposition.

5. L'exemple ci-dessous montre que toujours est-il que est à même de signaler la fin d'un commentaire métadiscursif:

[i] Armand, ça le dessaoule un peu, il écoute, il s'écarquille, il s'attendait pas! Son avocat à lui, c'est nettement le gabarit au-dessous, le bafouilleux quelconque, bigleux du dossier, endormeur debout des magistratures assises. Lubray, pardon! Quand il attaque sa chansonnette, ça devient l'Opéra tout de suite. Je l'ai assez dépeint, fignolé dans La Cerise, je vais pas encore vous le rabâcher. Toujours est-il qu'il a trouvé l'ouverture pour assumer ma défense.

(Frantext: A. Boudard, Les enfants de chour [1982], Paris, Gallimard, 1984, p. 225)

6. Sur le statut de toujours est-il que comme locution adverbiale, voir Hansen (2004 et 2008), et sur le figement des locutions prédicatives en général, voir Nøjgaard (I992: 132-133). Pour des études plus détaillées de toujours est-il que, voir Cadiot et al. (1985), Nguyen (1986), Fuchs (1988), Franckel (1989) et Nemo (2000).

7. ATILF (laboratoire Analyse et traitement informatique de la langue française). Base textuelle Frantext. En ligne à l'adresse suivante: http://www.frantext.fr/. 
ce qui va suivre par rapport à ce qui précède immédiatement (séquence intercalée). Deux configurations se dégagent alors selon que l'énoncé contenu dans la séquence intercalée est présenté sous la modalité du doute ou de l'hypothèse comme dans l'exemple [I] ou sous une modalité assertive catégorique comme dans l'exemple [2].

I. Lorsque la locution introduit ce dont le locuteur est certain en opposition à ce dont il est moins sûr ou ce qu'il ignore, elle signale la plus grande pertinence de ce qui va suivre par rapport à la séquence intercalée, en ce qui concerne la véracité des propos. Ainsi dans l'exemple [r], y a-t-il «discordance modale» dans le sens où il y a opposition entre l'incertitude exprimée par les interrogatives et la certitude du point de vue réaffirmé, et du fait de cette discordance modale, une différence de prise en charge énonciative. Toujours est-il que indique la forte prise en charge du locuteur relativement à ce qui suit, alors que ce qui précède immédiatement n'est pris en charge que très faiblement, d'où un effet de sens éventuellement concessif ${ }^{8}$.

2. Lorsqu'il n'y a pas de différence de degré de certitude entre la séquence intercalée et ce qui suit, toujours est-il que signale un simple contraste modal (exemple [2]), ce qui précède immédiatement est un jugement de valeur (ou un commentaire métadiscursif) interprété comme une digression que la locution vient clore; toujours est-il que marque la fin de la digression et signale la plus grande pertinence de ce qui va suivre par rapport à ce qui précède (séquence intercalée) en ce qui concerne non pas la véracité des propos mais en tant qu'objet de discours même, la différence de prise en charge étant alors perçue au niveau perlocutoire. Dans l'exemple [2], toujours est-il que indique que la visée perlocutoire globale du discours n'est pas de persuader de la pertinence du jugement de valeur porté sur les zazous en général par le locuteur, et que c'est le zazou rencontré dans l'autobus qui constitue l'objet de discours. La locution présente ainsi une valeur purement procédurale dans le sens où elle sert de guide à la lecture en assurant la progression dans la narration.

L'objectif du présent article est de conforter cette hypothèse d'une différence de prise en charge énonciative comme composante de la signification de toujours est-il que, et de confirmer l'existence de deux configurations. Par prise en charge, nous entendons ici le degré d'engagement du locuteur ${ }^{9}$, un engagement que nous décrivons sous la forme d'un continuum allant de la forte adhésion à la plus faible adhésion:

8. Toujours est-il que est d'ailleurs répertorié en tant que locution adverbiale concessive chez Morel (I996: I26) et Nøjgaard (I992: 484-485).

9. La notion de prise en charge est ici empruntée à Dendale et Coltier (2005), elle recouvre les liens de responsabilité et d'accord chez Nølke (1994 et 2006). Pour notre part, nous la mesurons au moyen $\mathrm{du}$ test de l'implication: toujours est-il que $p / d e$ toute évidence $p /$ tout compte fait $p$ impliquent $p$ (forte prise en charge), à première vue $p$ n'implique pas $p$ (faible prise en charge). Voir à ce sujet Lenepveu et Gosselin (2013) et Lenepveu (2014). Sur les différentes acceptions de la notion de prise en charge, voir Coltier et al. (2009). 
- dans un contexte de discordance, la prise en charge maximale marquée par toujours est-il que est associée par défaut à un haut degré de croyance du locuteur relativement au contenu propositionnel même si la locution peut bien sûr interagir avec d'autres marques de jugement (verbes et adverbes modaux). Ainsi, «toujours est-il que Pierre viendra peut-être demain» signifie la forte prise en charge par le locuteur de la simple probabilité de la venue de Pierre;

- lorsqu'il y a contraste modal, la forte prise en charge marquée par toujours est-il que est liée à un haut degré de pertinence: le cotexte droit immédiat constitue le propos principal, celui sur lequel le locuteur engage maximalement sa responsabilité.

Après avoir très rapidement rappelé dans une première section, la diversité des relations entre point de vue initial et segment de texte introduit avec toujours est-il que, nous nous proposons d'étudier les formes spécifiques à la séquence intercalée lorsque la différence de degré de prise en charge se manifeste sous la forme d'une discordance modale. Des interrogatives (éventuellement suivies d'un aveu d'ignorance), des modaux épistémiques, et un certain type de subordonnées concessives constituent typiquement la séquence intercalée ou la fin de cette séquence (Hansen, 2004; Buchi, 2007; Lenepveu, 2015) ${ }^{10}$. On veut montrer pourquoi et comment ces trois formes, morphologiquement et sémantiquement très différentes, jouent un rôle comparable dans la structuration du texte. En dehors de ces contextes spécifiques, il apparât parfois difficile de distinguer nettement entre les emplois de type I (exemple [I] - discordance modale) et les emplois de type 2 (exemple [2] - contraste modal). C'est pourquoi nous nous sommes intéressée aux traductions ${ }^{11}$ de cette locution, du français vers l'anglais, et de l'anglais vers le français. Cette confrontation nous a permis de confirmer: I) le rôle de toujours est-il que comme renforçateur de la prise en charge énonciative; et 2) l'existence de deux emplois distincts (discordance ou contraste modal), donnant lieu à deux traductions différentes.

Pour préciser la distinction opérée entre deux emplois, on remarque que le concept de discordance est utilisé par Morel (1996: 9 et I33) lorsqu'elle décrit la concession dite «logique» (Bien que P, Q). Il s'agit alors d'une opposition entre contenus normalement incompatibles. Ici, le concept de discordance modale est introduit pour traiter des cas où l'opposition porte non pas sur le contenu, mais sur le degré de validation à l'intérieur d'une même catégorie modale (en l'occurrence l'incertain et le certain dans le champ de la modalité épistémique). Quant au contraste modal, il renvoie à une opposition entre deux catégories modales (au sens de Gosselin, 20IO),

10. Voir également Hansen (2008: 200).

11. Pour une présentation critique des débats sur l'utilisation des corpus de traduction en sémantique, voir Celle (2006: 8-25). 
lorsque par exemple, à l'intérieur d'un fragment de texte aléthique ou épistémique, est inséré un passage relevant de modalités axiologiques et/ou appréciatives.

\section{Sur la relation entre point de vue initial et segment de texte introduit par toujours est-il que}

Le fait que l'énoncé introduit par toujours est-il que renvoie systématiquement à un segment textuel antérieur n'est pas toujours aisé à repérer pour deux raisons.

Tout d'abord, la séquence intercalée, entre le point de vue initial et l'énoncé introduit par toujours est-il, peut être très étendue (plusieurs pages), ce qui implique une recherche approfondie dans le contexte gauche de l'expression, une recherche que nous avons menée principalement à partir d'exemples initialement sélectionnés sur Frantext (M. Proust, L.-F. Céline, C. Simon) ou Arvensa Éditions (J. Verne) ${ }^{12}$. Par ailleurs, la relation entre le point de vue initial et la reprise de ce point de vue, introduite par toujours est-il, peut prendre des formes très diverses, si bien que le retour à un topique antérieur n'est pas toujours explicite ni immédiatement repérable. C’est donc un phénomène qui demande une lecture et une interprétation sémantique et pragmatique du texte.

Si nous n'avons pas rencontré de cas d'identité stricte, on peut dire que le point de vue est véritablement réaffirmé quand il peut s'interpréter comme une reformulation paraphrastique de ce qui vient d'être dit, ce qu'illustre parfaitement l'exemple [I] proposé dans notre introduction.

Mais le plus souvent, la relation va au-delà d'une relation de paraphrase, ce qu'on peut décrire, en partie, au moyen de la «théorie de la structure rhétorique» (Rhetorical Structure Theory - RST) (Mann et Thompson, I988; Mann et Taboada, 2005$\left.2012^{13}\right)$, le point de vue réaffirmé pouvant être interprété comme un développement (Élaboration), une justification (Justification), une confirmation (Confirmation), ou encore une conséquence (Résultat), du point de vue initial (Lenepveu, 20I5). Ainsi dans l'exemple [3] ci-dessous, le topique discursif que constitue «l'excellence du lard de morse en tant que combustible» se trouve repris et justifié par le segment de texte introduit par toujours est-il que:

[3] Le lard de morse, immédiatement utilisé dans le foyer ad hoc que portait l'Alaska, concurremment avec une faible proportion de houille, se trouva un combustible excellent. Son seul défaut était d'encrasser la cheminée et de nécessiter un nettoyage quotidien. Quant à son odeur, qui aurait sans doute impressionné désagréablement

12. À noter que chez J. Verne, la locution toujours est-il que se rencontre presque exclusivement dans les romans écrits en collaboration avec A. Laurie.

13. Voir le site consacré à la RST à l'adresse suivante: http://www.sfu.ca/rst. Nous reprenons les définitions des relations de la RST telles qu'elles sont proposées pour le français sur ce site. 
des passagers méridionaux, elle n'était pour un équipage suédois et norvégien qu'un inconvénient très secondaire.

Toujours est-il que, grâce à ce supplément, l'Alaska put rester sous vapeur jusqu'à la dernière heure, franchir rapidement, malgré les vents contraires, la distance qui le séparait encore des mers d'Europe et arriver, le 5 septembre, en vue du Cap-Nord de Norvège, sans même s'arrêter à Tromsoë, comme il l'aurait $\mathrm{pu}$, en cas de besoin; il poursuivit activement sa route, contourna la péninsule scandinave, repassa le Skagerrak et revint à son point de départ.

(Arvensa Éditions: J. Verne et A. Laurie, L'épave de Cyntbia [I866], chap. XX)

Toujours est-il que peut également introduire une information présupposée par le point de vue initial (exemple [4]) ou bien inférable à partir de ce point de vue (exemple [5]):

[4] On chercha en vain le philosophe norvégien. Une colique l'avait-elle saisi? Avait-il eu peur de manquer le train? Un aéroplane était-il venu le chercher? Avait-il été emporté dans une assomption? Toujours est-il qu'il avait disparu sans qu'on eût le temps de s'en apercevoir, comme un Dieu.

(M. Proust, Sodome et Gomorrhe [1922], chap. II, in À la recherche du temps perdu; cité par Le Bidois, 1952: 105)

[5] En revanche, j’avais de l'admiration pour Dudule le facteur des postes. Un échalas tout à fait conforme à celui créé par Jacques Tati dans Jour de fête. [...] Marquis notre clébard, mon pote le griffon, il accueillait pas mieux Dudule que les gendarmes. Tous les chiens de garde à la campagne montraient des crocs, s'excitaient après les uniformes. Je ne sais d'où leur venait cette aversion, elle était, en tout cas, tout à leur honneur. En contrepartie, ils ne manifestaient pas lerche d'affection non plus à l'égard des vagabonds. Carrément furibard, le Marquis, à la vision lointaine sur la route d'un trimardeur quelconque, même un simple journalier à la recherche d'embauche. Comportement à l'image de leurs maitres français moyens, ennemis aussi bien de la dictature que de l'anarchie. Toujours est-il que longtemps j’ai rêvé d'être facteur rural. Ce fut ma première vocation, et d'ailleurs je me demande encore si ce ne fut pas la seule... par la suite, j'ai jamais eu de but précis dans l'existence. Facteur, on était reçu partout, en général on vous supposait messager de bonnes nouvelles. On trinquait droite gauche.

(Frantext: A. Boudard, Mourir d'enfance [1995], Paris, Pocket, 1997, p. 24)

Enfin, avec l'exemple [2], nous avons vu que toujours est-il que pouvait signaler tout simplement la reprise de la narration en inscrivant un nouvel événement dans une suite d'événements.

Pour chaque cas de figure, c'est l'analyse du contenu de la séquence intercalée (ou de la fin de la séquence intercalée, c'est-à-dire du cotexte gauche immédiat) qui permettra de préciser dans quelles conditions l'interlocuteur interprétera celle-ci comme une digression, c'est-à-dire comme un changement temporaire de sujet (Milhe Poutingon, 20I2), ou dans nos termes, de topique discursif. 


\section{Sur la relation entre séquence intercalée et point de vue réaffirmé : étude du cotexte gauche immédiat}

\subsection{Analyse du cotexte gauche immédiat lorsqu'il y a discordance modale entre ce qui précède et ce qui suit la locution}

Lorsqu'il y a discordance modale ${ }^{14}$, nous observons, comme dit précédemment, que le cotexte gauche immédiat se réalise sous des formes syntaxiques bien spécifiques ${ }^{15}$. Il se présente en effet le plus souvent sous la forme:

I. d'une subordonnée concessive,

2. d'une question ou d'une série de questions totales ou partielles, suivies éventuellement d'un aveu d'ignorance,

3. d'une assertion modalisée au moyen notamment d'un verbe ou d'un adverbe de modalité épistémique de faible/forte probabilité (peut-être, sans doute, devoir, pouvoir, etc.), ou d'un verbe d'attitude propositionnelle non factif (je suppose que, etc.).

\subsubsection{Le cotexte gauche immédiat est une subordonnée concessive}

Lorsque le cotexte gauche prend la forme d'une concessive ${ }^{16}$, ce qui est fréquent dans les textes du XVIII ${ }^{e}$ ou XIX ${ }^{e}$ siècle, celle-ci s'apparente à deux types de concessives selon la classification de Fradin (1977: chap. I-II) ${ }^{17}$ : les concessives propositionnelles alternatives et les concessives extensionnelles hypothétiques non scalaires, des concessives dont le point commun est de poser comme indéniable/indiscutable la vérité de la proposition correspondant à la principale, aucune raison ne pouvant contrarier la vérité de cette proposition.

L'exemple [6] illustre le fonctionnement d'une concessive propositionnelle alternative en relation avec toujours est-il que $p$. La concessive exprime des alternatives

14. C'est à cet emploi que correspond la description de toujours est-il que du grammairien R. Le Bidois: «Toujours est-il (que) sert à introduire un fait ou un jugement que l'on pose comme certain, en vive opposition avec d'autres faits qui viennent d'être présentés sous le signe de l'hésitation, de l'incertitude ou de la probabilité» (Le Bidois, 1952: 104).

15. Voir aussi Hansen (2008: 200).

16. Celle-ci se présente généralement sur le plan syntaxique comme une proposition subordonnée à la proposition introduite par toujours est-il que, laquelle prend le statut de "principale». Mais la concessive peut dépendre grammaticalement de la phrase qui précède:

[ii] Mais le français, le latin, et le grec qu'on apprend aux enfants prennent trop de temps, soit qu'on les enseigne par de mauvais procédés, ou que ce soient les langues les plus difficiles du monde, ou encore que l'étude d'une langue quelconque soit ce qu'il y a de plus long et de plus aride pour les enfants; toujours est-il qu'à moins de facultés toutes spéciales, on sort du collège sans savoir ni le latin, ni le français, et le grec encore moins. (Frantext: G. Sand, Histoire de ma vie [1855], Paris, Calmann-Lévy, 1879, p. 368)

17. Voir aussi Muller (I996: chap. IV). 
et pour chacune des alternatives, elle signale que l'assertion correspondant à la principale est valide ${ }^{18}$ :

[6] Or, que l'on aime ou que l'on fasse peu de cas du style, toujours est-il que le parti adopté produit, sur tous, une très vive impression qui ne s'efface jamais du souvenir [...]. (Frantext: E. Viollet-Le-Duc, Entretiens sur l'architecture, Paris, A. Morel, t. 2, I872, p. 207)

La concessive est dans [6] de forme que $P$, en position détachée, sans lien anaphorique avec la principale, et contient deux propositions coordonnées par le ou exclusif. Mais l'hypothèse alternative peut être également marquée par soit que... ou encore, en l'absence d'une marque de subordination, par le seul ou exclusif. Par exemple:

[7] Soit qu'il désespérât du retour de la légitimité, soit qu'il fût ébranlé par d'augustes instances, soit enfin qu'il voulut fermer le gouffre des discordes civiles, toujours est-il que le vicomte de Montflanquin ne pensa pas devoir refuser plus longtemps l'appui de son nom au trône de juillet.

(Frantext: J. Sandeau, Sacs et parchemins, Paris, M. Levy, I85I, p. 6)

[8] Porthos comprit ou ne comprit pas, toujours est-il que, se laissant aller à sa colère:

- Monsieur, dit-il, vous vous ferez étriller, je vous en préviens.

(Frantext: A. Dumas père, Les trois mousquetaires [1844], Paris, Gallimard, 1996, p. 60)

L'exemple [9] illustre le fonctionnement des concessives extensionnelles hypothétiques en relation avec toujours est-il que, concessives qui supposent le parcours d'un domaine de quantification par une variable, la proposition introduite par toujours est-il que étant présentée comme vraie pour toute valeur de la variable. Dans cet exemple, c'est précisément la présence d'un indéfini antécédent d'un pronom relatif, sans fonction par rapport au verbe de la principale, qui, combinée à l'emploi du subjonctif, déclenche l'opération consistant à parcourir toutes les valeurs possibles sans que la valeur de vérité de la proposition correspondant à la principale ne s'en trouve affectée (Legoffic, 1993: 49I):

[9] Quelle que fût, du reste, son impression, toujours est-il qu'il s'arrêta, peu sûr de lui-même et de ses souvenirs, s'il en avait, au milieu de la contre-allée dans laquelle il s'était engagé.

(Frantext: J. Barbey d'Aurevilly, Les diaboliques [1874], Paris, Gallimard, 2003, p. 226)

18. Lorsque la subordonnée comporte plus de deux conjoints, elle s'apparente aux concessives extensionnelles (non scalaires) en s'interprétant comme le parcours d'un domaine de quantification, mais elle s'en distingue en ce qu'elles ne comportent pas de variable (Fradin, 1977: chap. I).

[iii] [...] que cette idée soit une chimère, une fée, un centaure, toujours est-il que nous l'avons, et, sous ce rapport, l'idée ne peut pas être fausse, elle ne peut pas ne pas être vraie, ou plutôt, à la rigueur, elle n'est ni fausse ni vraie.

(Frantext: V. Cousin, Cours de l'bistoire de la philosophie. Histoire de la philosophie morale au XVIII siècle, Paris, Pichon et Didier, 1829, t. 2, École sensualiste: Locke, p. 319) 
On note que les concessives extensionnelles hypothétiques attestées suggèrent une interprétation en termes d'identification plutôt qu'en termes d'intensité, et s'apparentent aux concessives extensionnelles non scalaires (Fradin, 1977; Soutet, 2008), des concessives dont le contenu propositionnel reste indéterminé, et dont la visée est là encore de renforcer la véracité de l'assertion correspondant à la principale $^{19}$. Alors qu'une concessive extensionnelle adjectivale supposant le parcours d'un domaine de quantification de degré et marquant le haut degré introduit l'idée d'une contradiction (Si malin qu'il fut, Goupil s'est fait prendre), et vise plutôt à contester la validité de la proposition contenue dans la subordonnée (il n'est pas si malin que ça) (Fradin, 1977: chap. IV), les deux types de concessives qui entrent en relation avec toujours est-il que servent à renforcer la validité de la principale.

\subsubsection{Le cotexte gauche immédiat est constitué d'interrogatives directes totales ou partielles}

Le cotexte gauche immédiat peut également se constituer d'une question ou d'une série de questions, une caractéristique définitoire de la question étant l'expression d'une incertitude par rapport à un contenu propositionnel (Anscombre et Ducrot, 1981; Therkelsen, 2009).

Lorsque le cotexte gauche est composé de questions, celles-ci prennent souvent la forme d'interrogatives directes ${ }^{20}$ totales ou partielles. Quand elles sont totales, elles se caractérisent par une inversion simple ou complexe du sujet (nominal ou pronominal), ou encore par la présence de la particule interrogative est-ce que. Ces interrogatives totales, qui représentent sur le plan sémantique des questions, n'expriment pas, dans ce contexte monologal, une demande d'information ou une requête, et s'interprètent comme des hypothèses: en posant une question, le locuteur présente l'assertion correspondante comme une explication possible de l'état de choses qui vient d'être évoqué (de manière implicite ou explicite). La question laisse les deux possibilités ouvertes ( $p$ et non $p$ ) mais elle marque la prédisposition du locuteur à admettre l'assertion sous-jacente de même polarité que la question, sans aller toutefois jusqu'à la prise en charge. Sur le continuum que pose Haillet (2007 et 2008) ${ }^{21}$ entre la question véritable (le locuteur cherche à s'informer) et la question rhétorique, elle

19. Et le fait que toujours est-il que puisse également entrer en relation avec une subordonnée en si factuelle, toujours détachée en position frontale, s'explique selon nous par la «mise en suspens» du contenu propositionnel de celle-ci :

[iv] [...] s'il y a peu de chance que ce principe fût jamais formulé de cette façon dans la famille de Catherine, toujours est-il que Catherine ne couchait pas avec Alexis.

$\overline{\text { (Frantext }}$ : E. Triolet, Le premier accroc coûte deux cents francs, Paris, Denoël, 1945, p. 163) À l'instar de Stage (1991), Monte (2009: 118) analyse ces subordonnées en si $P$ en tant que propositions extra-prédicatives non conditionnelles, mais reposant sur deux valeurs «inextricables» : une valeur de reprise due à leur antéposition, et une valeur énonciative de mise en suspens. Voir aussi Corminboeuf (2013) pour qui les si-constructions factuelles restent hypothétiques.

20. Des interrogatives indirectes sont également attestées, et dans ce cas, typiquement introduites avec un verbe d'ignorance (on ignore si, on ne sait pas trop si, on ne sait pas trop ce que, etc.) (Lenepveu, 2015).

21. Le refus du locuteur de prendre en charge la vérité de la proposition fonde aussi l'analyse de la question chez Rossari (2005) et Berrendonner (2005). 
constitue un intermédiaire, le locuteur étant plus ou moins prédisposé à admettre le point de vue correspondant à l'assertion dite sous-jacente. Il est ainsi relativement disposé à l'admettre dans l'exemple [ı], où la question est isolée:

[ro] - Eh bien, dites, monsieur, et ne nous faites pas languir ainsi.

Elle avait raison. Rouletabille le comprit-il? Toujours est-il qu'il racheta la lenteur de ses prolégomènes par la rapidité, la netteté, le saisissant relief avec lequel il retraça les événements de la nuit.

(Frantext: G. Leroux, Le parfum de la dame en noir, Paris, L’illustration, 1908, p. II7)

Dans le cas des questions alternatives, le locuteur admet que chacune des assertions correspondantes peut avoir une valeur explicative, et se montre disposé à accepter chacune des hypothèses sans en prendre une plus particulièrement en charge. Cette mise à distance reste implicite avec une série d'interrogatives totales juxtaposées (exemple [II]), elle est en revanche explicitée quand un aveu d'ignorance suit la ou les questions (exemple [I2]), et/ou lorsque l'hypothèse est attribuée à une tierce personne (exemple $\left.\left[\mathrm{I}_{3}\right]\right)$ :

[II] Est-ce que je vais mourir? Est-ce que je suis vieux? Est-ce que je deviens philosophe? Toujours est-il que le gouffre des choses éternelles me parait proche, si proche, que l'amour des choses temporelles et passagères me parait ridicule.

(Frantext: H.-F. Amiel, Journal intime de l'année 1866, Paris, Gallimard, 1959)

[I2] Avait-il eu la petite vérole, s'était-il brûlé avec le vitriol ou de la poudre, avait-il été défiguré par quelque horrible maladie? Mystère. Toujours est-il que cet homme avait un aspect repoussant et dur, même dans sa toilette, qui était d'une recherche exagérée et de mauvais goût.

(Frantext: P.-A. Ponson Du Terrail, Rocambole. Les drames de Paris [1859], Monaco, Éditions du Rocher, 1964, t. 2, p. 33)

[13] H. Drake avait-il été en effet foudroyé, comme l'affirmait Dean Pitferge; ou plutôt, ainsi que le soutint plus tard le médecin du bord, un vaisseau s'était-il rompu dans la poitrine du malheureux? Je n'en sais rien. Toujours est-il que nous n'avions plus sous les yeux qu'un cadavre.

(Arvensa Éditions: J. Verne, Une ville flottante [1871], chap. XXXIII)

Lorsque les questions sont partielles, nous retrouvons les mêmes structures syntaxiques (inversion simple et complexe du sujet, variante en est-ce que) ${ }^{22}$ :

[I4] Cette mascarade dura combien de temps? Quatorze ans, dit la légende; toujours est-il qu'à un moment l'empereur Héraclius parvint à réunir une immense armée et partit à la recherche de la Sainte Croix.

(Frantext: J.-K. Huysmans, L'oblat [1903], Paris, Crès, 1934, p. 4I)

22. Sauf que la seule présence du morphème interrogatif permet de maintenir l'ordre canonique sujet-verbe sans qu'on ait besoin de la variante en est-ce que. 
[I5] Qui formula le premier cette idée? C'est un point resté obscur et qui servit longtemps de thème aux discussions du docteur et de M. Bredejord; chacun prétendait la priorité. Sans doute ils l'eurent en même temps, car, à force de la cultiver, Erik devait en avoir saturé l'air ambiant. Toujours est-il qu'elle prit corps, qu'elle fut définitivement adoptée, et qu'au mois de septembre de l'année suivante, les trois amis, accompagnés d'Erik, s'embarquèrent à Christiania pour les États-Unis.

(Arvensa Éditions: J. Verne et A. Laurie, L'épave de Cynthia [I866], chap. VIII)

La question est suivie d'une réponse qui peut prendre la forme d'un aveu d'ignorance de la part du locuteur, ou d'une assertion attribuée à une tierce personne et/ou faiblement garantie par le locuteur mais, de nouveau, nous n'observons jamais d'engagement maximal explicite du locuteur.

\subsubsection{Le cotexte gauche immédiat: une modalisation atténuative}

Lorsque l'incertitude du locuteur s'exprime tout simplement par un verbe ou un adverbe de modalité, ceux-ci marqueront la probabilité épistémique, faible ou forte (peut-être, sans doute, certainement, etc.). Est exclue toute expression du haut degré de certitude (il est certain que, etc.):

[16] Peut-être M. Daudet tenait-il quelque chose de ce grand-oncle-là. Toujours est-il qu'il ne se connait à proprement parler aucune hérédité littéraire.

(Frantext: A. Daudet, Articles de journaux relatifs à Alphonse Daudet, I876-192I)

De même, un verbe d'attitude propositionnelle ne pourra jamais exprimer une conviction très forte de la part du locuteur:

[r7] Je suppose que les insomnies provoquant ma faiblesse physique actuelle y sont pour quelque chose. Toujours est-il que je viens d'errer dans la ville, absolument abruti de chagrin.

(Frantext: J.-P. Sartre, Lettres au castor et à quelques autres [1983], Paris, Gallimard, 20I0, vol. I (1926-1939), p. 18)

Dans le contexte présent, le point commun entre questions et assertions modalisées est qu'elles sont l'expression d'une incertitude, et que toujours est-il que peut se paraphraser par en tout cas ou quoi qu'il en soit, deux locutions qui, selon Rossari (1997, 2000) et Haillet (2000, 2007), ne remettent pas en cause la pertinence de l'énonciation (à la différence d'une locution comme de toute façon) mais l'affaiblissent, et qui, dans le contexte de discordance modale étudié, maintiennent rétroactivement l'incertitude du locuteur quant à ce qui vient d'être dit (Rossari, 1997: 86). En tout cas signale une «position de repli» (Haillet, 2007: 54-58) sur ce qui est sûr, assuré, par opposition à ce dont on est moins sûr; et quoi qu'il en soit, en tant que forme tronquée d'une concessive propositionnelle alternative (Rossari, 1997: 104), signifie que la réalisation ou non de l'état de choses évoqué à la fin de la séquence intercalée ne change rien à la validité de la proposition qui suit $(\approx$ que l'état de chose $X$ soit ou que l'état de chose $X$ ne soit pas). Les deux paraphrases mettent en évidence la faible 
prise en charge par le locuteur de ce qui vient d'être dit alors que toujours est-il que explicite l'engagement maximal du locuteur relativement au segment de texte qui suit. La locution présente ainsi, en aval, une portée cadrative et s'apparente ici aux cadratifs à portée véridictionnelle (Charolles, 1997; Sarda et Charolles, 2012) en signalant que le jugement à venir est fortement pris en charge par le locuteur.

\subsection{Analyse du cotexte gauche immédiat lorsqu'il y a contraste modal entre la séquence intercalée et le segment de texte qui suit toujours est-il que}

En l'absence de discordance modale entre le segment de texte qui constitue la séquence intercalée et le segment de texte introduit par la locution toujours est-il que, celle-ci signale la plus grande pertinence de ce qui va suivre par rapport à la séquence intercalée en tant qu'objet (topique) de discours, tel l'extrait du texte de Queneau (exemple [2]), avons-nous dit, où la locution donne l'instruction selon laquelle l'attention de l'interlocuteur doit se focaliser sur la suite des événements introduite après toujours est-il que. La locution joue alors simultanément un rôle de cadratif «rétroactif» dans le sens où elle marque la clôture de la séquence intercalée, et demande à lire cette séquence comme une digression, laquelle est constituée d'un jugement de valeur. Toujours est-il que se prête alors beaucoup moins bien à la stratégie de repli opérée avec en tout cas, mais la locution est en revanche remplaçable par quoi qu'il en soit ${ }^{23}$. La séquence intercalée reste affectée d'un moindre degré de prise en charge lié non plus à un faible degré de certitude, mais à un moindre degré de pertinence en tant qu'objet de discours. Soit l'exemple [I8] à titre d'illustration: ${ }^{24}$

[I8] Il avait passé là des après-midi épouvantables, malheureux comme les pierres. Sans compter qu'un fâcheux souvenir lui restait de ce temps-là. C'était ce jour où, il ne savait pourquoi, Fragonard lui avait confié son veston à garder. C'était grand chez lui. Avec des arbres partout, des futaies, des allées sinueuses. On était pas les uns sur les autres. Toujours est-il qu'il s'était trouvé seul à un moment, assis dans l'herbe, loin du court et de la bande. Alors, il n'avait pas résisté au désir de fouiller dans le portefeuille de Fragonard.

$\approx$ quoi qu'il en soit/?en tout cas il s'était trouvé seul à un moment, assis dans l'herbe, loin du court et de la bande. Alors, il n'avait pas résisté au désir de fouiller dans le portefeuille de Fragonard ${ }^{24}$.

(Frantext: R. Guérin, L'apprenti [1946], Paris, Gallimard, 1949, p. 95)

23. Les deux locutions toujours est-il que et quoiqu'il en soit sont rapprochées chez Morel (1996: I26) et Nøjgaard (1992: 485). Une étude systématique des différences avec les locutions quasi paraphrastiques en tout cas et quoi qu'il en soit serait évidemment très utile.

24. Du fait de leur fonction structurante, on peut opérer un rapprochement entre toujours est-il que et bref qui, dans son emploi interphrastique, en position antéposée, joue, dans les termes de Bastian et Hammer (2005: 175-177), un rôle «d'opérateur d'ouverture» et sert à «vectoriser» la progression de la narration ou de l'argumentation. Mais toujours est-il que ne prend ni la valeur abréviative ni la valeur résomptive de bref(Schnedecker, 1992). 
Reste que la différence que nous établissons entre contraste modal et discordance modale n'est pas suffisante pour identifier un segment de texte comme étant digressif ou non, notamment lorsque la séquence intercalée relève du discours assertif (assertions modalisées). Ainsi, dans l'exemple ci-dessous, la discordance modale, marquée à deux reprises par la tournure impersonnelle il me semble que (soulignée ici) à la fin de la séquence intercalée, n'exclut pas d'interpréter celle-ci comme une digression, alors qu'une série de questions relatives aux circonstances de l'état de choses évoqué (exemple [I]) sera difficilement perçue comme telle ${ }^{25}$ :

[19] On m'a fait franchir le portail et on m'a chargé dans une ambulance où il y avait des gendarmes. Charlotte est montée avec moi. À travers le portail ouvert, je voyais des types sortir des bureaux de l'institution, cinq ou six types, en file indienne et les mains sur la tête, encadrés par Coccioli et Haymann et deux ou trois policiers qui tenaient des revolvers. Ensuite est apparue l'espèce de bonzesse chauve. Quelqu'un l'aidait à marcher. Il m'a semblé qu'elle était blessée et qu'elle pleurait. Je ne me rappelle pas avoir vu de clients de l'institution. Plus tard, il me semble que certains ont élevé des protestations parce qu'ils avaient été brutalisés. Toujours est-il que les gendarmes ont fermé les portes de l'ambulance et j’ai replongé dans le coton. Alors pour ce qui est de l'action violente, c'est terminé, mais je suppose que je dois dire encore ce qu'il est advenu des protagonistes.

(Frantext: J.-P. Manchette, Que d'os!, Paris, Gallimard, 1976, p. 216)

Une recherche des équivalences possibles de toujours est-il que en anglais nous autorise cependant à confirmer l'existence de deux emplois pour cette locution. Dans un premier temps, nous observons les différentes traductions anglaises de la locution prise dans les romans de la littérature française du XIX et du XX siècle (M. Proust, L.-F. Céline, J. Verne, C. Simon), puis nous partons de l'œuvre originale complète de C. Dickens, avec pour corpus les traductions françaises issues des Classiques illustrés eBooks (lci-eBooks). Il apparait que si l'observation du passage du français vers l'anglais conforte l'hypothèse d'une différence de prise en charge énonciative marquée par toujours est-il que quel que soit le contexte, c'est le passage de l'anglais au français qui permet de poser une véritable équivalence entre toujours est-il que et une marque de modalité signalant un haut degré de certitude (it is certain/certain it is). Cette équivalence ne valant que pour le seul contexte de discordance, elle confirme ainsi l'existence de deux emplois pour toujours est-il que.

\section{Du français vers l'anglais: deux types de marqueurs en anglais pour toujours est-il que}

Sur la trentaine d'occurrences de toujours est-il que repérées dans notre corpus de littérature française du XIX ${ }^{e}$ et du XX $X^{e}$ siècle (M. Proust, L.-F. Céline, C. Simon,

25. Voir à cet égard Rossari (2005) qui montre que l'énonciation d'une question et celle d'une assertion communiquant le même type d'information n'autorisent pas les mêmes enchaînements discursifs monologiques. 
J. Verne), il s'avère que les expressions choisies dans les traductions anglaises expriment bien une composante de la signification de toujours est-il que qui est de signaler une différence de prise en charge ${ }^{26}$. Deux types de marqueurs sont à distinguer:

I. Des expressions concessives ou susceptibles d'un emploi concessif (Bourquin, 1984; Guimier, 1988; Ranger, 1998) sont à même de traduire toujours est-il que et sont disponibles pour les deux contextes dégagés (discordance ou contraste modal): anyway/anyhow/in any case/at any rate/either way/be that as it may/whatever the truth of the matter/however/however that may be/but/etc ${ }^{27}$.

- Dans un contexte de discordance (exemple [20]), ces expressions signalent la plus faible pertinence quant à la véracité du contenu de la séquence intercalée (ou de la fin de la séquence intercalée), et par là sa plus faible prise en charge par le locuteur:

[2oa] Pendant plus d'une semaine qu'elle a réfléchi, et elle m’a laissé bien tranquille... Elle avait dû en confier deux mots à sa mère de mes accès... Toujours est-il qu'elles insistaient moins pour me garder... "Ça y est que je me disais moi, ça va aller! Me voilà libre...» (Frantext: L.-F. Céline, Voyage au bout de la nuit [1932], Paris, Gallimard, 2000, p. 456)

[2ob] She thought for more than a week and didn't bother me all that time... She must have said a word or two to her mother about my fits... Anyway, they didn't seem so eager to hang on to me anymore... "Good deal!" I said to myself. "It's going to be all right! Free at last! ...”

(L.-F. Céline, Journey to the End of the Night, R. Manheim (trad.) et W. T. Vollmann (postface), New York, New Directions, 2006, p. 393)

- Dans un contexte de contraste modal, elles indiquent la plus faible pertinence de la séquence intercalée en tant qu'objet de discours, et par là encore sa plus faible prise en charge par le locuteur. Soit l'exemple [2] redonné en [2I]:

[2ra] Cette fois-là je n'avais dans mon voisinage que des hommes, dont une sorte de zazou au col démesuré et qui portait autour de son feutre mou une espèce de tresse au lieu de ruban. Comme si on ne devrait pas envoyer tous ces gars-là dans des camps de travail. Pour relever les ruines par exemple. Celles des anglo-saxons surtout. De

26. Nos conclusions seraient bien sûr à confirmer par l'étude de gros corpus de traduction mais les résultats obtenus sont d'ores et déjà significatifs.

27. Dans la classification de Fraser (2009b), des adverbiaux comme anybow, in any case, in any event, etc. ne font pas partie des "Topic Orientation Markers» (to change to topic, by the way, back to my original point, to return to my point, etc.) mais des «Attention Markers» (ok, so, so good, alright, well, well then, etc.). 
mon temps on était camelot du roy, et pas swing. Toujours est-il que ce garnement se permet tout à coup d'engueuler un ancien combattant, un vrai, de la guerre de $\mathbf{1 4}$-18. Et ce dernier qui ne riposte pas! (Frantext: R. Queneau, Exercices de style [1947], Paris, Gallimard, I963, p. I30)

[2rb] That time I was surrounded entirely by men, one of whom was a sort of teddy boy whose neck was of inordinate length and who was wearing a felt hat with a kind of plait round it instead of a ribbon. They ought to send all creatures of that sort off to labour camps. To repair the war damage. That caused by the anglo-saxons, especially. In my day we were Young Royalists, not Rock'n Rollers. At any rate this young object suddenly makes so bold as to start abusing an ex-service man, a real one, from the 1914 war. And he doesn't even answer back!

(R. Queneau, Exercises in style, B. Wright (éd. et trad.), New York, New Directions, 2009, p. 137)

2. Des marqueurs d'affirmation forte font valoir la validité de la proposition qui suit, et se distinguent ainsi des expressions concessives qui - comme on vient de le voir - servent à atténuer la partie qui précède le point de vue réaffirmé. Ainsi, l'expression the fact remains that signale la plus forte prise en charge «de ce qui suit», prise en charge liée à la véracité du discours (discordance modale) ou liée à la pertinence de l'objet de discours (contraste modal). Pour illustration, la comparaison de deux traductions différentes d'un même extrait de l'ouvrage Un amour de Swann confirme le choix possible entre un marqueur concessif et the fact remains that dans un contexte de discordance ${ }^{28}$. Soit l'extrait original:

[22a] C'est aussi du respect qu'inspirait à Odette la situation qu'avait Swann dans le monde mais elle ne désirait pas qu'il cherchât à l'y recevoir. Peut-être sentait-elle qu'il ne pourrait pas y réussir, et même craignaitelle, que rien qu'en parlant d'elle, il ne provoquât des révélations qu'elle redoutait. Toujours est-il qu'elle lui avait fait promettre de ne jamais prononcer son nom. La raison pour laquelle elle ne voulait pas aller dans le monde, lui avait-elle dit, était une brouille qu'elle avait eue autrefois avec une amie qui, pour se venger, avait ensuite dit du mal d'elle.

(Frantext: M. Proust, Du côté de chez Swann [1913], $2^{\mathrm{e}}$ partie, in À la recherche du temps perdu, Paris, Gallimard, 1962, p. 242)

et deux traductions attestées:

28. Des exemples attestent également de la disponibilité de l'expression the fact remains that lorsqu'il y a contraste modal. 


\section{- /the fact remains that/}

[22b] There was also the respect with which Odette was inspired by the thought of Swann's social position, although she had no desire that he should attempt to secure invitations for herself. Perhaps she felt that such attempts would be bound to fail; perhaps, indeed, she feared lest, merely by speaking of her to his friends, he should provoke disclosures of an unwelcome kind. The fact remains that she had consistently held him to his promise never to mention her name. (M. Proust, Swann's Way, C. K. Scott Moncrieff (trad.), New York, Holt, 1922, p. 449)

\section{- / in any case/}

[22c] Respect, too, was what was inspired in Odette by Swann's position in society, but she did not want him to try to secure invitations for her. Perhaps she felt that he might not be successful, or was even afraid that merely by talking about her he would prompt revelations that she dreaded. In any case, she had made him promise never to utter her name.

(M. Proust, The Way by Swann's, C. Prendergast (éd.) et L. Davis (trad.), Londres, Penguin Books, 2003, p. 244)

Parmi les marqueurs d'affirmation forte, on a pu également relever, dans un contexte de discordance, des adverbes ou des expressions modales (one thing is sure, certainly, definitely):

[23a] [...] depuis ce moment, chaque matin, il s'en allait par le tramway à Paris soi-disant à la recherche d'un emploi de représentant.

Il en avait assez de l'usine, qu'il disait, il voulait «représenter». Il s'est peut-être donné du mal pour en trouver une de représentation, faut être juste mais enfin toujours est-il qu'il l'a pas trouvée.

Un soir il est rentré de Paris plus tôt qu'à l'habitude. J'étais encore au jardin moi, en train de surveiller les abords du grand bassin. Il est venu me retrouver là pour me dire deux mots.

(Frantext: L.-F. Céline, Voyage au bout de la nuit [1932], Paris, Gallimard, 2000, p. 447)

[23b] And every morning from then on he took the streetcar in to Paris, to look for a job as a salesman.

He was «sick of factories», he said, he wanted to be a salesman. I've got to be fair, he may have knocked himself out looking for a salesman's job, but he certainly didn't find one.

(L.-F. Céline, Journey to the End of the Night, R. Manheim (trad.) et W. T. Vollmann (postface), New York, New Directions, 2006, p. 385) 

sur cette apparente équivalence entre une locution assurant une fonction connective comme toujours est-il que et une marque de modalité ${ }^{30}$. C'est afin d'en préciser les conditions que nous avons adopté la démarche qui a consisté à partir de l'œuvre originale complète de C. Dickens et à identifier les contextes favorisant le choix de la locution toujours est-il que dans une traduction française.

\section{De l'anglais vers le français}

Sur 43 occurrences de toujours est-il que repérées dans les traductions des Classiques illustrés eBooks (lci-eBooks), nous avons relevé I3 contextes dans lesquels toujours est-il que ne semble pas traduire un marqueur lexical ou grammatical particulier, tandis que 2I occurrences traduisent des connecteurs concessifs (be that as it may/be it as it might/but be it what it may/be that how it might/however/however that was/anyhow/still/but), et que 9 occurrences traduisent des marqueurs d'affirmation forte (it is certain that/(but) certain it is that/it's undeniably/indeed). On remarque encore que si les 43 contextes correspondent à la structure discursive imposée par toujours est-il que, la locution n'est utilisée pour traduire un marqueur de haut degré de certitude que s'il y a discordance modale avec le segment de texte qui précède immédiatement. Les conditions d'équivalence sont alors les suivantes:

I. un point de vue initial,

2. une séquence intercalée,

3. un point de vue réaffirmé entretenant une discordance modale avec la séquence intercalée.

Les exemples [24] et [25] illustrent l'équivalence possible de certain it is that/it is certain that et toujours est-il que dans cette configuration, avec à la fin de la séquence intercalée une des formes spécifiques étudiées à la section 3.I (questions, hypothèses alternatives, assertions modalisées), et soulignée ici:

[24a] As Mr Quilp folded his note (which was soon written: being a very short one) he encountered the gaze of the small servant. He looked at her, long and earnestly.

"How are you?" said the dwarf, moistening a wafer with horrible grimaces.

The small servant, perhaps frightened by his looks, returned no audible reply; but it appeared from the motion of her lips that she was inwardly repeating the same form of expression concerning the note or message.

"Do they use you ill here? is your mistress a Tartar?" said Quilp with a chuckle.

29. Je dois cette remarque à Shirley Carter-Thomas.

30. Grieve (1996) ne mentionne pas ce type d'équivalence. Outre la tournure the fact remains that, l'auteur donne les équivalents anglais suivants: anyway, all the same, even so, nevertheless, nonetheless, whatever the case may be, but (Grieve, 1996: 485) ainsi que anyhow (Grieve, 1996:20). Ne figurent pas still et however qui sont en revanche donnés comme équivalents potentiels de n'empêche que et reste que (Grieve, 1996: 22-26). 
In reply to the last interrogation, the small servant, with a look of infinite cunning mingled with fear, screwed up her mouth very tight and round, and nodded violently. Whether there was anything in the peculiar slyness of her action which fascinated Mr Quilp, or anything in the expression of her features at the moment which attracted his attention for some other reason; or whether it merely occurred to him as a pleasant whim to stare the small servant out of countenance; certain it is, that he planted his elbows square and firmly on the desk, and squeezing up his cheeks with his hands, looked at her fixedly.

(lci-eBooks: C. Dickens, The Old Curiosity Shop [I840-I84I], II, chapter XII)

[24b] Le billet fut promptement écrit; il était très court. Tout en le pliant M. Quilp rencontra le regard de la petite servante. Il examina longtemps et curieusement cette jeune fille.

«Comment vous trouvez-vous ici?» dit le nain en mâchant un pain à cacheter avec d'horribles grimaces.

La petite servante, effrayée peut-être par cet examen, ne put articuler une réponse intelligible; mais le mouvement de ses lèvres permettait de comprendre qu'elle répétait intérieurement sa même phrase au sujet d'une carte ou d'une lettre.

«Est-ce qu'on vous traite mal, ici? Votre maîtresse n'est-elle pas un vrai cosaque?» dit Quilp d'un ton caressant.

À cette dernière question, la petite servante, avec un regard très fin mêlé de crainte, serra fortement sa bouche arrondie, et secoua vivement la tête.

Soit qu'il y eût dans cette vivacité de mouvement quelque chose qui plût à M. Quilp, ou que l'expression qu'avaient prise les traits de la petite servante fixât son attention pour un autre motif; soit tout simplement qu'il voulût s'amuser à lui faire perdre contenance, toujours est-il qu'il posa carrément ses coudes sur le pupitre, et, pressant ses joues entre ses mains, se mit à la dévisager.

(lci-eBooks: C. Dickens, Le magasin d'antiquités [A. Des Essarts (trad.), I876], II, chap. XIII)

L'exemple [25] met particulièrement bien en évidence le rôle comparable que jouent ces formes dans la structuration du texte puisqu'une hypothèse alternative se trouve traduite en français par une série de questions suivie d'un aveu d'ignorance:

[25a] But Mr Titre Barnacle was a buttoned-up man, and consequently a weighty one. All buttoned-up men are weighty. All buttoned-up men are believed in. Whether or no the reserved and never-exercised power of unbuttoning, fascinates mankind; whether or no wisdom is supposed to condense and augment when buttoned up, and to evaporate when unbuttoned; it is certain that the man to whom importance is accorded is the buttoned-up man. Mr Titre Barnacle never would have passed for half his current value, unless his coat had been always buttoned-up to his white cravat. (lci-eBooks: C. Dickens, Little Dorrit [1855-I857], livre II, Riches, chap. XII)

[25b] Mais il faut dire que M. Tenace Mollusque était un homme boutonné jusqu'au menton, et par conséquent un homme de poids. Tout homme boutonné jusqu'au 
menton est considéré comme un homme profond. Est-ce parce qu'il y a dans cette faculté de se déboutonner qu'il se réserve d'exercer ou non, quelque chose qui en impose à l'humanité en général? Ou bien ne se figure-t-on pas que la sagesse se condense et s'accroit lorsqu'elle est si solidement renfermée dans une double rangée de boutons, sans quoi elle risque de s'évaporer? Je l'ignore; mais toujours est-il que l'homme toujours boutonné jusqu'au menton est celui qui donne la plus haute idée de son importance. M. Tenace Mollusque aurait perdu une bonne moitié de sa valeur courante si son habit n'avait pas été constamment boutonné jusqu’à sa cravate blanche. (lci-eBooks: C. Dickens, La petite Dorrit [P. Lorrain (éd.) et W. Little Hughes (trad.), I894], livre II, Richesse, chap. XII)

Par ailleurs, lorsque toujours est-il que ne traduit pas un item lexical ou grammatical particulier, les conditions d'équivalence restent identiques, et nous retrouvons les formes spécifiques à la séquence intercalée :

[26a] It wouldn't be an unhappy life, Amy. It would be the life I am fitted for. Whether by disposition, or whether by circumstances, is no matter, I am better fitted for such a life than for almost any other.

(lci-eBooks: C. Dickens, Little Dorrit [1855-1857], livre II, Riches, chap. XIV)

[26b] Ce ne serait pas une existence malheureuse pour moi, Amy; c'est celle qui me convient le mieux. Que ma nature ou le concours des circonstances l'ait voulu ainsi, peu importe: toujours est-il que cette existence-là me conviendrait mieux que toute autre.

(lci-eBooks: C. Dickens, La petite Dorrit [P. Lorrain (éd.) et W. Little Hughes (trad.), I894], livre II, Richesse, chap. XIV)

Le fait que dans un contexte de discordance, toujours est-il que puisse être équivalent à un marqueur de modalité épistémique ${ }^{31}$ confirme ainsi l'existence de deux emplois pour cette locution: l'emploi identifié par le grammairien Le Bidois (toujours est-il que introduit ce dont on est sûr par opposition à ce dont on est moins sûr), et l'emploi de toujours est-il que comme simple marqueur de fin de digression.

\section{Conclusion}

Si la locution toujours est-il que indique un retour au topique initial, et marque également la clôture de la séquence intercalée, simultanément, elle précise le statut énonciatif de cette séquence intercalée relativement au point de vue initial et au segment de texte introduit par toujours est-il que. La séquence intercalée est en effet affectée d'un moindre degré de prise en charge lié à un moindre degré de pertinence : elle ne constitue pas le propos principal sur lequel le locuteur engage maximalement sa responsabilité. Cet affaiblissement du degré de prise en charge énonciative se manifeste sous deux formes:

31. Sur la fonction de connexion d'éléments modalisateurs en français, voir entre autres Vion (2006). 
I. une discordance modale opposant l'incertitude (exprimée par des subordonnées concessives, des modaux épistémiques, des interrogatives, des verbes d'ignorance) à la certitude du point de vue réaffirmé;

2. un contraste modal, généralement entre un discours servant à décrire une situation, et un ou plusieurs jugement(s) de valeur intercalé(s) à titre de digression.

Le passage du français à l'anglais a montré que toujours est-il que pouvait se traduire en anglais par des marqueurs d'affirmation forte pour signifier la plus forte prise en charge (the fact remains that, one thing is sure, certainly, definitely, etc.) du segment de texte qui suit, ou bien par des marqueurs concessifs pour signifier la plus faible prise en charge du segment de texte qui précède (at any rate, anyway, either way, be that as it may, whatever the truth of the matter, etc.). La démarche inverse a confirmé l'équivalence possible entre toujours est-il que et un marqueur de modalité épistémique exprimant un haut degré de certitude (certain it is/it is certain), les conditions de cette équivalence étant:

I. une discordance modale avec la séquence qui précède,

2. un point de vue initial qui se trouve réaffirmé.

Lorsque l'équivalence est exclue (parce que les conditions ne sont pas réunies), toujours est-il que devient un véritable marqueur de fin de digression, et s'apparente à donc en tant que marqueur de reprise (Leroy, 2005) ou encore aux expressions du type pour en revenir à ce que je disais, pour en revenir à nos moutons, etc.

\section{Références}

Anscombre, J.-C. et Ducrot, O. 1981. Interrogation et argumentation. Langue française $52: 5^{-22}$.

Bastian, S. et Hammer, F. 2005. Bref et en somme marqueurs de glose. In A. Steuckardt et A. Niklas-Salminen (éd.), Les marqueurs de glose. Aix-en-Provence: Publications de l'université de Provence: I7I-I89.

Berrendonner, A. 2005. Question et mémoire discursive. In C. Rossari, A. BeaulieuMasson, C. Cojocariu et A. Razgouliaeva (éd.), Les états de la question. Québec: Éditions Nota Bene: I47-I7I.

Blumenthal, P. 1980. La syntaxe du message. Application au français moderne. Tübingen: M. Niemeyer.

Bourquin, G. 1984. Discours de langue et discours de parole: les énoncés concessifs. RANAM I7: 7-33.

BuchI, É. 2007. Sur la trace de la pragmaticalisation de l'adverbe toujours («Voyons toujours l'apport de la linguistique historique»). Langue française I54: IIO-I25.

Cadiot, A., Ducrot, O. et NGuyen, T.-B. 1985. Sous un mot, une controverse : les emplois pragmatiques de toujours. Modèles linguistiques 7 (2): 105-I24.

Celle, A. 2006. Temps et modalité: l'anglais, le français et l'allemand. Berne: P. Lang. 
Charolles, M. 1997. L'encadrement du discours : univers, champs, domaines et espaces. Cabiers de recherche linguistique 6: $\mathrm{I}-73$.

Charolles, M. 20i6. Quelle place réserver aux marqueurs de topique de discours dans les taxinomies de marqueurs de discours? Document présenté à la rencontre du I2-I3 septembre 2016 dans le cadre du projet Labex TransferS «Marqueurs de changement de topique de discours».

Coltier, D., Dendale, P. et De Brabanter, P. 2009. La notion de prise en charge : mise en perspective. Langue française $162: 3^{-27}$.

Corminboeuf, G. 2013. Factualité et conditionnalité. In C. NorÉn, K. JonAsson et H. NøLKe (éd.), Modalité, évidentialité et autres friandises langagières: mélanges offerts à Hans Kronning à l'occasion de ses soixante ans. Berne - Berlin - Bruxelles: P. Lang: 4I-6o.

Dendale, P. et Coltier, D. 2005. La notion de prise en charge ou de responsabilité dans la théorie scandinave de la polyphonie linguistique. In J. Bres, P.-P. Haillet, S. Mellet, H. Nølke et L. Rosier (éd.), Dialogisme et polyphonie: approches linguistiques. Bruxelles: De Boeck: I25-I40.

Fradin, B. 1977. Les concessives extensionnelles en français moderne. Thèse de doctorat. Université Paris 8.

FRANCKEL, J.-J. 1989. Étude de quelques marqueurs aspectuels du français. Genève: Droz.

Fraser, B. 2009a. Towards a Theory of Discourse Markers. In K. Fischer (éd.), Approaches to Discourse Particles. Amsterdam: Elsevier: I89-204.

Fraser, B. 2oogb. Topic Orientation Markers. Journal of Pragmatics 4I (5): 892-898.

Fuchs, C. 1988. Encore, déjà, toujours: de l'aspect à la modalité. In N. Tersis et A. KinM (éd.), Temps et aspects [Actes du colloque CNRS, Paris, 24-25 octobre 1985]. Louvain - Paris : Peeters - SELAF : I35-I48.

Gosselin, L. 20ıо. Les modalités en français. Amsterdam - New York: Rodopi.

Grieve, J. 1996. Dictionary of Contemporary French Connectors. New York - Londres: Routledge.

Guimier, C. 1988. Syntaxe de l'adverbe anglais. Villeneuve-d'Asq: Presses universitaires de Lille.

Haillet, P.-P. 200o. Or, en tout cas, de toute façon: contribution de la «théorie de l'argumentation dans la langue» à la pratique de la traduction. In D. Delas (éd.), Traduire I. Cergy-Pontoise - Paris : Centre de recherche texte-histoire (CRTH) - Les belles lettres: I87-209.

Haillet, P.-P. 2007. Pour une linguistique des représentations discursives. Bruxelles: De Boeck.

Haillet, P.-P. 2008. Réalité du locuteur: vers une typologie des points de vue. In M. Birkelund, M.-B. M. Hansen et C. Norén (éd.), L'énonciation dans tous ses états: mélanges offerts à Henning Nølke à l’occasion de ses soixante ans. Berne : P. Lang : 47-62.

Hansen, M.-B. M. 2004. La polysémie de l'adverbe toujours. Travaux de linguistique 49:39-55.

Hansen, M.-B. M. 2008. Particles at the Semantics/Pragmatics Interface: Synchronic and Diachronic Issues. A Study with Special Reference to the French Phasal Adverbs. Amsterdam New York - Paris: Elsevier.

LE BidoIs, R. I952. L'inversion du sujet dans la prose contemporaine (I900-1950). Paris: Éditions d'Artray. 
Le Goffic, P. 1993. Grammaire de la phrase française. Paris: Hachette.

Lenepveu, V. 20I4. Aspect de dicto et structuration informationnelle dans les textes. Lingvistice Investigationes 37 (I): 129-155.

Lenepveu, V. 20I5. Toujours est-il que ( $p$ ) ou le retour à un topique antérieur. Discours I7: I-26. En ligne à l'adresse suivante: http://discours.revues.org/907I.

Lenepveu, V. et Gosselin, L. 2013. Les marqueurs d'aspect de dicto: à première vue, à y regarder de plus près, au bout du compte, tout compte fait... In C. NorÉn, K. Jonasson et H. NøLKe (éd.), Modalité, évidentialité et autres friandises langagières: mélanges offerts à Hans Kronning à l'occasion de ses soixante ans. Berne - Berlin - Bruxelles: P. Lang: 227-269.

Leroy, S. 2005. Sécurité, donc sûreté. L'emploi de donc comme marqueur de glose. In A. Steuckardt et A. Niklas-Salminen (éd.), Les marqueurs de glose. Aix-en-Provence: Publications de l'université de Provence: I59-170.

Mann, W. C. et Taboada, M. 2005-20I6. Rhetorical Structure Theory (RST) Web Site. Accessible à l'adresse suivante: http://www.sfu.ca/rst.

Mann, W. C. et Thompson, S. A. 1988. Rhetorical Structure Theory: Toward a Functional Theory of Text Organization. Text 8 (3): 243-28I.

Martin, R. 1987. Langage et croyance. Les univers de croyance dans la théorie sémantique. Bruxelles: P. Mardaga.

Milhe Poutingon, G. 20I2. Poétique du digressif. La digression dans la littérature de la Renaissance. Paris : Classiques Garnier.

Monte, M. 2009. Si marqueur d'altérité énonciative dans les si $P$ extraprédicatives non conditionnelles. Langue française 163 : 99-II9.

Morel, M.-A. 1996. La concession en français. Paris - Gap: Ophrys.

Muller, C. 1996. La subordination en français: le schème corrélatif. Paris: A. Colin.

Nemo, F. 2000. Enfin, encore, toujours entre indexicalité et emplois. In A. ENGLEBERT, M. Pierrard, L. Rosier et D. Van Raemdonck (éd.), Actes $d u$ XXII congrès international de Linguistique et de Pbilologie romanes (Bruxelles, 23-29 juillet 1998). Tübingen: M. Niemeyer. Vol. 7: Sens et fonctions: 499-511.

Nguyen, T.-B. 1986. Toujours est-il. Revue Romane 2I (2): 192-207.

NøjgaARD, M. 1992-I995. Les adverbes français. Essai de description fonctionnelle. Historiskfilosofiske Meddelelser 66. Copenhague: Munksgaard. 3 vol.

NøLke, H. 1994. Linguistique modulaire: de la forme au sens. Louvain - Paris: Peeters - Société pour l'information grammaticale.

NøLke, H. 2006. Pour une théorie linguistique de la polyphonie: problèmes, avantages, perspectives. In L. PERRIN (éd.), Le sens et ses voix. Dialogisme et polyphonie en langue et en discours. Recherches linguistiques 28. Metz: université Paul-Verlaine: 243-269.

RANGER, G. 1998. Les constructions concessives en anglais: une approche énonciative. Paris - Gap: Ophrys.

Rossari, C. 1997. Les opérations de reformulation. Analyse du processus et des marques dans une perspective contrastive français-italien. Berne - Berlin - Paris: P. Lang [ $2^{\mathrm{e}}$ éd.].

Rossari, C. 2000 . Connecteurs et relations de discours: des liens entre cognition et signification. Nancy: Presses universitaires de Nancy. 
Rossari, C. 2005. Les questions totales: une évocation de l'état des connaissances de l'autre. In C. Rossari, A. Beaulieu-Masson, C. Cojocariu et A. Razgouliaeva (éd.), Les états de la question. Québec: Éditions Nota Bene: 173-189.

SARDA, S. et Charolles, M. (éd.) 20I2. Travaux de linguistique: Les adverbiaux prépositionnels position, fonction et portée 64 . Bruxelles: Duculot.

Schnedecker, C. 1992. Bref, un marqueur d'opération résumante? In M. Charolles et A. Petitjean (éd.), Le résumé de texte: aspects linguistiques, sémiotiques, psycholinguistiques et automatiques. Recherches linguistiques I7. Metz - Paris: université de Metz - Klincksieck: 28-48.

Soutet, O. 2008. Des concessives extensionnelles aux concessives simples. Contribution à l'étude de la genèse sémantique et historique des locutions conjonctives concessives du français. LINX 59: II5-I32.

StAGE, L. 199I. Analyse syntaxique et sémantique de la conjonction si dans les propositions factuelles. Revue romane 26 (2): 163-205.

Therkelsen, R. 2009. Comment identifier une question polyphonique? Langue française I64: II3-I22.

VAn Dijk, T. A. 1976. Sentence Topic and Discourse Topic. Papers in Slavic Philology I : 49-6I.

VION, R. 2006. Modalisation, dialogisme et polyphonie. In L. PERRIN (éd.), Le sens et ses voix. Dialogisme et polyphonie en langue et en discours. Recherches linguistiques 28. Metz: université Paul-Verlaine: I05-I25.

\section{CEuvres littéraires traduites}

CÉLine, L.-F. 1966. Death on the Installment Plan. R. Manheim (trad.). New York: New Directions.

CÉLIne, L.-F. 2006. Journey to the End of the Night. R. Manheim (trad.) et W. T. Vollmann (postface). New York: New Directions.

Proust, M. 1922. Swann's Way. C. K. Scott Moncrieff (trad.). New York: Holt.

Proust, M. 2003. The Way by Swann's. C. Prendergast (éd.) et L. Davis (trad.). Londres: Penguin Books.

Queneau, R. 2009. Exercises in Style. B. Wright (éd. et trad.). New York: New Directions.

Simon, C. 1989. The Georgics. J. Fletcher et B. Fletcher (trad.). Londres - New York: J. Calder - Riverrun Press.

\section{Éditions en ligne}

Littérature classique numérique: Arvensa Éditions.

Les classiques illustrés eBooks: lci-eBooks.

\section{Base de données}

ATILF (laboratoire Analyse et traitement informatique de la langue française). Base textuelle Frantext. En ligne à l'adresse suivante: http://www.frantext.fr/. 